\title{
IDENTIFICATION OF REPLICATOR-MUTATOR MODELS
}

\author{
Paola Falugi *,1 Laura Giarré ** \\ * DSI, Università di Firenze, Firenze, Italy. \\ falugi@dsi.unifi.it \\ ** DIAS, Università di Palermo, Palermo, Italy. \\ giarre@unipa.it
}

\begin{abstract}
The complexity of biology literally calls for quantitative tools in order to support and validate biologists intuition and traditional qualitative descriptions. In this paper, the Replicator-Mutator models for Evolutionary Dynamics are validated/invalidated in a worst-case deterministic setting. These models analyze the DNA and RNA evolution or describe the population dynamics of viruses and bacteria. We identify the Fitness and the Replication Probability parameters of a genetic sequences, subject to a set of stringent constraints to have physical meaning and to guarantee positiveness. The conditional central estimate is determined in order to validate/invalidate the model. The effectiveness of the proposed procedure has been illustrated by means of simulation experiments while tests on real data are under concern.
\end{abstract}

Keywords: Biomedical Systems, Evolutionary Dynamics, Nonlinear systems, Positive systems, Uncertain Dynamical Systems

\section{Introduction}

In this paper we consider the problem of identifying the model of evolutionary dynamics. Darwinian evolution is based on three fundamental principles, reproduction, mutation and selection, which describe how populations change over time and how new forms evolve out of old ones.

The aim is validate/invalidate in a worst-case deterministic setting the Replicator-Mutator models. The model is well-known in its structure and is in general positive and nonlinear.

\section{Replicator-Mutator and Quasispecies Models}

There are numerous mathematical descriptions of the resulting evolutionary dynamics. In (Page

\footnotetext{
1 Partially supported by MIUR-PRIN "Robustness and optimization Techniques for high performance control systems".
}

and Nowak, 2002) apparently very different formulations that have been studied in literature are presented as part of a single unified framework. In particular the two most common models are the Quasispecies Equation (see (Eigen et al., 1989)) of molecular evolution and the Replicator-Mutator Equation (see (Hadeler, 1981), (Eigen and Schuster, 1979)). Eigens quasispecies model was developed as a framework for studying the dynamics of replicating DNA and RNA macromolecules. Later it has been used to describe the population dynamics of more advanced biological systems such as viruses and bacteria. It considers an individual as a finite sequence of elementary building blocks or bases. The quasispecies model has proved to be very fruitful as a reasonably general model of Darwinian evolution that incorporates selection mutation and a genome length larger than one. It has been an excellent theoretical framework to ap- 
proach RNA virus dynamics. Theoretical as well as empirical research has been used for medical or pharmaceutical strategies to face viruses.

As well described in (Komanova, 2004), the Replicator-Mutator equation appears in three different contexts in biology:

\section{Population gentics:}

see (Hadeler, 1981), (Hofbauer and Sigmund, 1998). Here $x_{i}$ denotes the relative frequencies of allels at the time of mating.

Autocatalytic reaction networks:

see (Stadler and Schuster, 1992),(Schuster and Stadler, 2003). Here $x_{i}$ are the concentrations of molecules, RNA of DNA, which are capable of self-replications.

Population language learning:

see (Nowak et al., 2001),(Komanova, 2004). Here $x_{i}$ are the relative abundance of individuals which use a specific grammar.

Let us focus on a specific context and $x_{i}$ denotes the relative abundance of a genetic sequence $i$ in a population. The fitness, $f_{i}$ of this sequence is determined by its replication rate. The average fitness of the population is given by $\bar{f}=\sum_{i} f_{i} x_{i}$. We consider $n$ genetic sequences. According to (Nilsson, 2000), the matrix $Q$ has been introduced to describe how mutations affect a population. In particular $q_{i j}$ is defined as the the probability that replication of genome $j$ gives genome $i$ as offspring. For perfect copying accuracy $Q$ equals the identity matrix. Mutations give rise to the off-diagonal elements in $q_{i j}$. Below, we report an explicit expression for $Q$ in terms of the copying fidelity as given by (Nilsson, 2000),

$$
q_{i j}=q^{\nu}\left(\frac{1-q}{q}\right)^{h_{i j}}
$$

where $h_{i j}$ is the Hamming distance between genomes $j$ and $i$, and $\nu$ is the genome length and $q$ is the copying accuracy. The Hamming distance $h_{i j}$ is defined as the number of positions where genomes $j$ and $i$ differ.

The Quasispecies Equation describes the dynamics of the genomes $x_{i}$ :

$$
\dot{x}_{i}=\sum_{j=1}^{n} x_{j} f_{j} q_{i j}-\bar{f} x_{i}
$$

This is a nonlinear quadratic model exhibiting bilinear dependence in the parameters. It can be equivalently recast in a more concise form as follows:

$$
\dot{x}=\left(Q \operatorname{diag}(f)-\left(f^{T} x\right) I\right) x
$$

where the state variable is $x=\left[x_{1} \ldots x_{n}\right]^{T}$ and $f=\left[f_{1} f_{2} \ldots f_{n}\right]^{T}$. Putting together the quasi- species equation with the Lotka-Volterra equation, that takes into account the fact that the reproductive fitness $f_{i}$ is function of the abundance of other species and is $f_{i}(x)$, in (Nowak et al., 2001) or (Wilke et al., 2001) it has been derived the Replicator-Mutator equation:

$$
\dot{x}_{i}=\sum_{j=1}^{n} x_{j} f_{j}(x) q_{j i}-\bar{f} x_{i}
$$

Clearly, depending on how the fitness is related to $x_{i}$, various model have been developed in literature. In particular, according to (Komanova, 2004), we consider a polynomial expansion:

$$
f_{j}=w_{j}+\sum_{k=1}^{n} \gamma_{j k} x_{k}+\ldots \quad 1 \leq j \leq n
$$

If we keep only the linear terms, then

$$
f=w+\Gamma x
$$

where $\Gamma=\left[\gamma_{i j}\right]$ and $w=\left[w_{1} \ldots w_{n}\right]^{T}$.

Then, the replicator-mutator equation can be recasted as

$$
\begin{aligned}
\dot{x} & =\left(Q(\operatorname{diag}(w)+\operatorname{diag}(\Gamma x))-\left(w^{T} x+x^{T} \Gamma^{T} x\right) I\right) x \\
& =\left(Q(\operatorname{diag}(w)+\operatorname{diag}(x) \Gamma)-\left(w^{T} x\right) I-x^{T} \Gamma^{T} x I\right) x
\end{aligned}
$$

Clearly, if $\gamma_{i j}=0$ we obtain the quasispecies models given in (3), and if $q_{i j}=1$ for $i=j$ and $q_{i j}=0$ for $j \neq i$ we obtain the replicator model (see (Hofbauer and Sigmund, 1998)).

\section{Model Parametrization}

Quasispecies Models in (3) enjoys the following properties:

P.1 Starting with $\sum_{i} x_{i}(0)=1$ then $\sum_{i} x_{i}(t)=$ $1, \forall t>0$. This can be expressed as: $\mathbf{1}^{T} x=1$ where $\mathbf{1}^{T}$ denotes a row-vector of ones. This means that the state variables take value in the unitary simplex $\Sigma$.

P.2 The system is positive: starting from any initial condition $x(0)>0, x(t)>0 \forall t>0$.

P.3 $q_{i j} \in[0,1]$

P.4 $f_{i} \geq 0$

From the above assumptions, we can derive some constraints on the parameters $q_{i j}$ and $f_{i}$.

\section{Lemma 1}

For the unitary simplex $\Sigma$ to be positively invariant conditions P.3, P.4 and

$$
\mathbf{1}^{T} Q=1^{T}
$$

need to be satisfied. 
The models ( 3 ) and ( 7 ) are overparameterized. In the next Lemma, it is shown that $f$ is defined up to simultaneous translation of its entries.

\section{Lemma 2}

Let $\tilde{Q}$ and $\tilde{f}$ satisfy

$$
\begin{gathered}
\tilde{f}=f+\lambda \mathbf{1} \\
\tilde{f}_{i}>0 \quad \forall i \\
\tilde{Q}=[Q \operatorname{diag}(f)+\lambda I)][\operatorname{diag}(f)+\lambda I)]^{-1}
\end{gathered}
$$

for a real number $\lambda$, and $\tilde{x}(t)$ denotes the solution of equation (3) where $f$ and $Q$ are replaced by $\tilde{f}$ and $\tilde{Q}$. Then, starting from the same initial conditions,

$$
\tilde{x}(0)=x(0)
$$

the systems has the same dynamics:

$$
\tilde{x}(t)=x(t) \quad \forall t \geq 0
$$

\section{Identification}

Despite the identification methodologies are well established in many application fields, their use in the parameters estimation of the evolutionary systems is quite rare. Most of the literature in this area deals with the modeling of the systems, without a rigorous validation and/or data-based parameter estimation. In some applications, the solution is based on statistical approaches like the Maximum Likelihood Principle (see (Bielawski and Z., 2003) for a recent survey, or the parameter estimation based on time-series (i.e. (Bonhoeffer et al., 2002)).

On the other hand, if we consider literature on identification of positive systems there are some results for linear systems. In this case the positive systems are compartmental. Some results are based on statistical approaches (Benvenuti et al., 2002)) with solution based on the Maximum Likelihood Principle, other results are based on the interval literature, (Kieffer and Walter, 2004). For nonlinear positive systems (that are not compartmental), not much can be found in the identification literature.

Almost all these contributions assume a statistical description of the noise and are mainly devoted to point estimation while little attention is devoted to the computation of confidence regions for the parameter estimates although they are important for the assessment of the model quality. Conversely, the assumption of Unknown But Bounded (U.B.B.) noise (see (Milanese and Vicino, 1991) for an extensive survey) naturally rises the issue of computing the Feasible Parameter Set (FPS).

\section{Regressor Form}

In order to identify the parameters $q_{i j}$ and $f_{i}$, first we must recast the model in a regressor form. The model in (3) and (7) is formed by $n=2^{\nu}$ continuous-time differential equations. Assume that we discretize it with a standard firstorder Euler approximation with sampling time $T$. Then the discrete-time system becomes

$$
\begin{aligned}
x_{i}(t+1)= & \sum_{\substack{j=1 \\
j \neq i}}^{n} \alpha_{j}^{i} x_{j}(t)+\sum_{k=1}^{n} \bar{\beta}_{k}^{i} x_{k}(t) x_{i}(t)+ \\
& +\sum_{\substack{j=1 \\
j \neq i}}^{n} \sum_{\substack { k=1, k \neq j \\
k \neq i \\
\begin{subarray}{c}{n \\
j{ k = 1 , k \neq j \\
k \neq i \\
\begin{subarray} { c } { n \\
j } }\end{subarray}}^{n} \delta_{j, k}^{i} x_{k}(t) x_{j}(t)+ \\
& +x_{i}(t) \sum_{j=1}^{n} \sum_{\substack{k=1 \\
k \neq j}}^{i} \mu_{j, k}^{i} x_{j}(t) x_{k}(t) \\
& +e_{i}(t) \quad \\
= & g(x(t))+e_{i}(t) \\
& \text { for } i=1, \ldots, n
\end{aligned}
$$

where

$$
\begin{aligned}
\alpha_{i}^{i}= & 1+T q_{i i}\left(w_{i}+\gamma_{i i}\right) \\
\alpha_{j}^{i}= & T q_{i j}\left(w_{j}+\gamma_{j j}\right) \\
\bar{\beta}_{i}^{i}= & \alpha_{i}^{i}-T\left(w_{i}+\gamma_{i i}\right) \\
\bar{\beta}_{k}^{i}= & 1+T\left(q_{i i} w_{i}-w_{k}-\gamma_{k k}\right)+ \\
& +T\left(\gamma_{k i} q_{i k}+\gamma_{i k} q_{i i}-q_{i k} \gamma_{k k}\right) \\
\delta_{j, k}^{i}= & T\left(\gamma_{j k} q_{i j}+\gamma_{k j} q_{i k}-\gamma_{k k} q_{i k}-\gamma_{j j} q_{i j}\right) \\
\mu_{j, k}^{i}= & T\left(\gamma_{j j}-\gamma_{j k}+\gamma_{k k}-\gamma_{k j}\right)
\end{aligned}
$$

We note that in equation (14) we have already exploited the condition P.1. In fact if $\mathbf{P . 1}$. is satisfied $\left(\sum_{j} x_{j}=1\right)$, we have that:

$$
x_{j}(t)=x_{j}(t) \sum_{k=1}^{n} x_{k}(t)
$$

and

$$
x_{i}(t) x_{j}(t)=x_{i}(t) x_{j}(t) \sum_{k=1}^{n} x_{k}(t)
$$

giving raise to the elimination of the following $2 n$ terms: $x_{i}, x_{j}^{2}$, for $j \neq i$ and $x_{i} x_{j}^{2}$.

Notice also that in equation (14) we have considered a process error $e_{i}$ on which we make the following assumption:

E.1 The error term is U.B.B. :

$$
|e|_{\infty} \leq \epsilon
$$

Moreover, in order to guarantee the invariance of the simplex $\Sigma$, the error term is such that:

E.2

$$
\sum_{i} e_{i}=0 .
$$

E.3

$$
0 \leq g\left(x^{2}(t)\right)+e_{i}(t) \leq 1 \text {. }
$$

Moreover we assume that the state variables are measurable without any further noise error; we have output variables: 


$$
y_{i}=x_{i}
$$

The system can be recast in a regressor-like form as follows. Let us consider a single measurement.

$$
Y(t+1)=\Phi(t) \Theta+E(t)
$$

where $Y(t)=\left[\begin{array}{llll}y_{1}(t) & y_{2}(t) & \ldots & y_{n}(t)\end{array}\right]^{T}, e(t)=$ $\left[e_{1}(t) e_{2}(t) \ldots e_{n}(t)\right]^{T}$,

$$
\begin{gathered}
\text { for } i=1, \ldots, n \\
y_{i}(t+1)=\phi_{i}(t) \theta^{i}+e_{i}(t) \\
\Phi(t)=\left[\begin{array}{lllll}
\phi_{1}(t) & 0 & 0 & \ldots & 0 \\
0 & \phi_{2}(t) & 0 & \ldots & 0 \\
\vdots & \vdots & \vdots & \vdots & \\
0 & 0 & & \ldots & \phi_{n}(t)
\end{array}\right], \\
\Theta=\left[\begin{array}{llll}
\theta^{1} & \theta^{2} & \ldots & \theta^{n}
\end{array}\right]^{T}
\end{gathered}
$$

Each $\theta^{i}$ is composed by the $n^{2}$ parameters defined in (15) that have to be determined:

$$
\begin{aligned}
\theta^{i}= & {\left[\begin{array}{llllll}
\alpha_{1}^{i} & \ldots & \alpha_{i-1}^{i} & \alpha_{i+1}^{i} & \ldots & \alpha_{n}^{i} \ldots \bar{\beta}_{1}^{i} \ldots \bar{\beta}_{n}^{i} \\
& \delta_{1,2}^{i} \ldots \delta_{n,(n-1)}^{i} \mu_{1,2}^{i} & \ldots & \mu_{n,(n-1)}^{i}
\end{array}\right]^{T} }
\end{aligned}
$$

and each row component $\phi_{i}(t)$ of the regressor is a vector defined at time $t$ as

$$
\phi_{i}^{T}=\left[\begin{array}{l}
y^{(i)} \\
y_{i} Y \\
z^{(i)} \\
y_{i} h
\end{array}\right]
$$

where

$$
\begin{aligned}
& y^{(i)}=\left[\begin{array}{lllll}
y_{1} & \ldots & y_{i-1} & y_{i+1} & y_{n}
\end{array}\right]^{T}, \\
& y_{i} Y=\left[\begin{array}{llllll}
y_{1} y_{i} \ldots & y_{i-1} y_{i} & y_{i}^{2} & y_{i+1} y_{i} \ldots & y_{n} y_{i}
\end{array}\right]^{T} \text {, } \\
& z^{(i)}=\left[z_{1}^{(i)} \ldots z_{\frac{(n-2)(n-1)}{2}}^{(i)}\right]^{T}
\end{aligned}
$$

and

$$
h=\left[h_{1} \ldots h_{\frac{(n)(n-1)}{2}}\right]^{T}
$$

being $z_{j}^{(i)}=y_{k}^{(i)} y_{l}^{(i)}$ for $k=1, \ldots, n-2 ; l=k+$ $1, \ldots, n-1$, and $h_{j}=y_{k} y_{l}$ for $k=1, \ldots, n-$ $1, l=k+1, \ldots, n$. Note that each $i$-regressor $\phi_{i}$ is formed by $n^{2}$ terms $\left(n+(n-1)+\frac{(n-2)(n-1)}{2}+\right.$ $\left.\frac{n(n-1)}{2}=n^{2}\right)$.

\section{Experiment setup and constraints}

For the sake of simplicity, let us consider now the quasispecies models, when $\gamma_{i j}=0$.

In order to guarantee experimental identifiabil$i t y$, the system is initialized from $m$ different (randomly generated) initial conditions. For each initial condition, the identification experiment is composed by $N * n$ measurements, and the overall data is a vector of $n * N * m$ elements:

$$
\mathcal{Y}=\left[\begin{array}{l}
Y(t+1) \\
Y(t+2) \\
\vdots \\
Y(t+N * m)
\end{array}\right]
$$

$$
\Psi=\left[\begin{array}{l}
\Phi(t) \\
\Phi(t+1) \\
\vdots \\
\Phi(t+N * m-1)
\end{array}\right]
$$

$$
\mathcal{E}=\left[\begin{array}{l}
E(t) \\
E(t+1) \\
\vdots \\
E(t+N * m-1)
\end{array}\right]
$$

so that:

$$
\mathcal{Y}=\Psi \Theta+\mathcal{E}
$$

In order to guarantee the structural identifiability we notice that at each time $t$ the original parameters to be identified $\left(q_{i j}\right.$ and $\left.f_{i}\right)$ are $n^{2}+n$. Recalling that the $f_{i}$ parameters are subject to the constraint (9) and that the $q_{i j}$ parameters are subject to $n$ constraints corresponding to eq. (8). The effective number of free parameters to be determined is $n^{2}+n-1-n=n^{2}-1$. In the $\Theta$-space, we need to estimate $n(2 n-1)$ parameters subject to $n$ constraints obtained from the relation $(8)$ and $(n-1)(n-1)$ constraints obtained transforming relation (9) into the new space. Then, the free parameters to be estimated are $2 n^{2}-n+n+(n-1)(n-1)=n^{2}-1$. The problem is well posed. The new-constraints in the $\Theta$-space have been simply determined, and their expression is reported hereafter in terms of its components. The $n$ constraints (8) are equivalent to the $n$ equality constraints

$$
\begin{gathered}
\text { for } j=1, \ldots, n \\
\sum_{\substack{k=1 \\
j \neq k}}^{n} \alpha_{j}^{k}+\bar{\beta}_{j}^{j}=1
\end{gathered}
$$

The constraint $(9)$ is equivalent to the $(n-1)(n-$ 1) equality constraints:

$$
\begin{aligned}
& \text { for } j=2, \ldots, n \\
& \quad \text { for } k=2, \ldots, n \\
& \quad \bar{\beta}_{j}^{1}-\bar{\beta}_{1}^{1}-\bar{\beta}_{j}^{k}+\bar{\beta}_{1}^{k}=0
\end{aligned}
$$

Moreover, we recall that the model must satisfy further disequality constraints given in P.3 and P.4. In the $\Theta$-parameter space, correspondingly, we can impose that:

$$
\begin{aligned}
& \text { for } j=1, \ldots, n \\
& \text { for } i=1, \ldots, n \\
& \alpha_{j}^{i} \geq 0 i \neq j
\end{aligned}
$$


Under the assumption of bounded error given in E.1 it is possible to define an extended Feasible Parameter Set (FPS) as:

$$
\Omega=\{\Theta:|\mathcal{Y}-\Psi \Theta| \leq \epsilon\}
$$

Let as define $\mathcal{M}$ the set of all the feasible parameters subject to the constraints (30), (31) and (32):

$$
\mathcal{M}=\{\Theta:(31),(30) \text { and (32) are satisfied }\}
$$

The following identification problem have been considered and solved.

Problem : Least Squares Conditional Central Estimate

$$
\Theta^{*}=\arg \min _{\Theta \in \mathcal{M}}\|\mathcal{Y}-\Psi \Theta\|_{2}
$$

The computational burdens of this problem amounts to solving one constrained Quadratic Programming (QP) Problem.

\section{Numerical Example}

The effectiveness of the proposed Procedure obtained by solving Problem 1 and Problem 2 is now illustrated on some numerical examples. For sake of clarity we report hereafter only the results corresponding to the simple case of a genetic sequence obtained considering $\nu=2$ in equation (1) that gives raise to $n=4$ state variables. More complex genetic sequences have been tested. The system is sampled with $T=0.01 \mathrm{~s}$. In the identification experiment, carried out with $N=151$ measurements using $\ell_{\infty}$ noise bounds with $\epsilon=0.015$, the system is initialized with $m=60$ different randomly generated initial conditions $x(0)$. The overall data are $N m n=36240$.

The real parameters are:

$$
\begin{gathered}
Q=\left[\begin{array}{llll}
0.1600 & 0.2400 & 0.2400 & 0.3600 \\
0.2400 & 0.1600 & 0.3600 & 0.2400 \\
0.2400 & 0.3600 & 0.1600 & 0.2400 \\
0.3600 & 0.2400 & 0.2400 & 0.1600
\end{array}\right] \\
F=\left[\begin{array}{l}
9.5013 \\
2.3114 \\
6.0684 \\
4.8598
\end{array}\right]
\end{gathered}
$$

Solving Problem we obtain the $(2 n-1) n=28$ $\theta_{i}$ components of $\Theta$.

We carry out the inversion procedure and we find that $h=2$. We set $\tilde{f}_{2}=1.6384$. The obtained Conditional Central Estimate are :

$$
Q^{*}=\left[\begin{array}{llll}
0.1620 & 0.2825 & 0.1921 & 0.4120 \\
0.2387 & 0.1221 & 0.3535 & 0.2186 \\
0.2393 & 0.3925 & 0.1961 & 0.2196 \\
0.3600 & 0.2029 & 0.2583 & 0.1498
\end{array}\right]
$$

$$
F^{*}=\left[\begin{array}{l}
9.4354 \\
1.6384 \\
7.7782 \\
4.7299
\end{array}\right]
$$

Validating the model on a different set-up (different initial condition and noise) the resulting dynamics evolution is reported in Fig. 1. In Fig. 2 the difference of true and estimated evolution is reported.

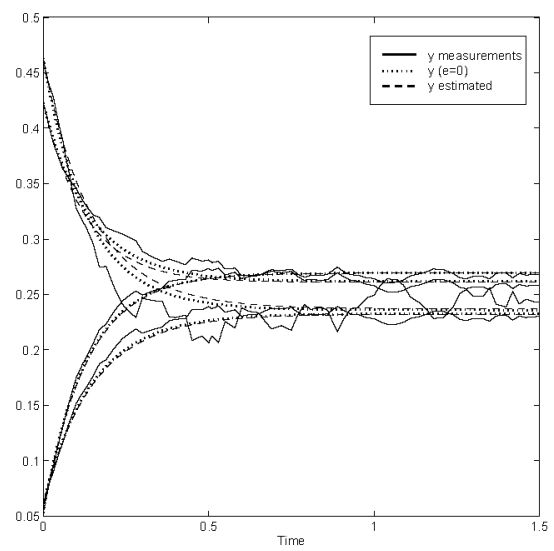

Fig. 1. Validation: evolution comparison.

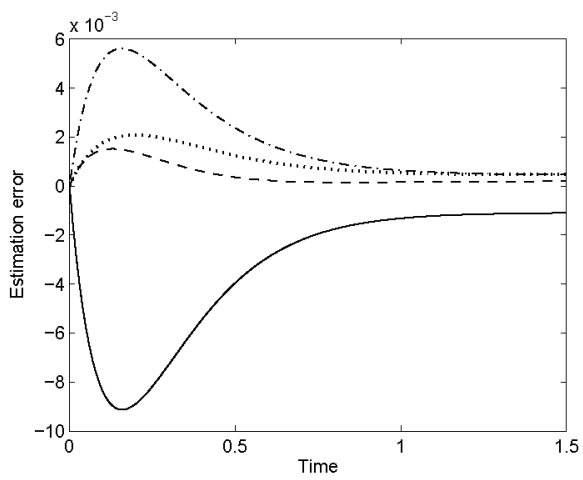

Fig. 2. Validation: error evolution.

Given the estimated $\Theta^{*} \in \mathcal{M}$, we should invert relations (15) in order to find a solution in the original space of parameters. Proposition in (Falugi and Giarré, 2006) gives solution and conditions to the determination of the unique inversion.

We note that the determination of the central estimate is very good. This is true also for simulation examples with greater $n$.

\section{REFERENCES}

Benvenuti, L., A. De Santis and A. Farina (2002). On model consistency in compartmental systems identification. Automatica 38, 1969 1976 . 
Table 1 . True and identified $\theta_{i}$

\begin{tabular}{|c|c|c|}
\hline & true $\theta$ & identified $\theta$ \\
\hline$\theta_{1}$ & 0.0055 & 0.0064 \\
\hline$\theta_{2}$ & 0.0146 & 0.0120 \\
\hline$\theta_{3}$ & 0.0175 & 0.0151 \\
\hline$\theta_{4}$ & 0.9202 & 0.9210 \\
\hline$\theta_{5}$ & 0.9921 & 0.9969 \\
\hline$\theta_{6}$ & 0.9545 & 0.9614 \\
\hline$\theta_{7}$ & 0.9666 & 0.9724 \\
\hline$\theta_{8}$ & 0.0228 & 0.0225 \\
\hline$\theta_{9}$ & 0.0218 & 0.0221 \\
\hline$\theta_{10}$ & 0.0117 & 0.0121 \\
\hline$\theta_{11}$ & 0.9087 & 0.9044 \\
\hline$\theta_{12}$ & 0.9806 & 0.9803 \\
\hline$\theta_{13}$ & 0.9430 & 0.9448 \\
\hline$\theta_{14}$ & 0.9551 & 0.9558 \\
\hline$\theta_{15}$ & 0.0228 & 0.0225 \\
\hline$\theta_{16}$ & 0.0083 & 0.0081 \\
\hline$\theta_{17}$ & 0.0117 & 0.0123 \\
\hline$\theta_{18}$ & 0.9147 & 0.9106 \\
\hline$\theta_{19}$ & 0.9866 & 0.9865 \\
\hline$\theta_{20}$ & 0.9490 & 0.9510 \\
\hline$\theta_{21}$ & 0.9611 & 0.9620 \\
\hline$\theta_{22}$ & 0.0342 & 0.0340 \\
\hline$\theta_{23}$ & 0.0055 & 0.0052 \\
\hline$\theta_{24}$ & 0.0146 & 0.0149 \\
\hline$\theta_{25}$ & 0.9128 & 0.9091 \\
\hline$\theta_{26}$ & 0.9847 & 0.9850 \\
\hline$\theta_{27}$ & 0.9471 & 0.9495 \\
\hline$\theta_{28}$ & 0.9592 & 0.9605 \\
\hline
\end{tabular}

Bielawski, J. and Yang Z. (2003). Maximum likelihood methods for detecting adaptive evolution after gene duplication. Journal of Structural and Functional Genomics 3(1-4), 201 212.

Bonhoeffer, S., A. D. Barbour and R. J. De Boer (2002). Procedures for reliable estimation of viral fitness from time-series data. Proceedings: Biological Sciences 269(1503), 1887 1893.

Eigen, M. and P. Schuster (1979). The Hypercycle. A Principle of Natural Self-Organisation. Springer- Berlin.

Eigen, M., Mccaskill and J. Schuster (1989). The molecular quasispecies. Adv. Chem. Phys. $75,149-263$.

Falugi, P. and L. Giarré (2006). Parameter bounded estimation for quasispecies models of molecular evolution. In: Proceedings of the IFAC SYSID.

Hadeler, K. P. (1981). Stable polymorphisms in a selection model with mutation. 41, $1-7$.

Hofbauer, J. and K. Sigmund (1998). Evolutionary Games and Replicator Dynamics. Cambridge University Press, Cambridge.

Kieffer, M. and E. Walter (2004). Guaranteed nonlinear state estimator for cooperative systems. Numerical Algorithms 37, 187-198.

Komanova, N.L. (2004). Replicator-mutator equation, universality property and populations dynamics of learning. Journal of Theoretical Biology pp. 227-239.

Milanese, M. and A. Vicino (1991). Optimal estimation theory for dynamic systems with set membership uncertainty: an overview. Automatica 27, 997-1009.

Nilsson, M. (2000). Mathematical Models Of Molecular Evolution. PhD thesis. Götenborg University.

Nowak, M.A., N.L. Komarova and P. Niyogi (2001). Evolution of universal grammar. Science 291, 114-118.

Page, K. and M.A. Nowak (2002). Unifying evolutionary dynamics. J. theor. Biol. 219, 92-98.

Schuster, P. and P.F. Stadler (2003). Networks in molecular evolution. Complexity pp. 34-42.

Stadler, P.F. and P. Schuster (1992). Mutation in autocatalytic reaction networksan analysis based on perturbation theory. J. Math. Biol. 30, 597-632.

Wilke, C., Ronnewinkel C. and Martinetz T. (2001). Dynamic fitness landscapes in molecular evolution. Phisycs Reports-Review section of Physics letters 349(5), 395-446. 